\title{
ABC of Sexually Transmitted Diseases
}

\section{COMPLICATIONS OF COMMON GENITAL INFECTIONS AND INFECTIONS IN OTHER SITES}

\begin{tabular}{|c|c|c|c|}
\hline \multirow[b]{2}{*}{ Complications } & \multicolumn{3}{|c|}{ Infection } \\
\hline & Gonococcal & $\begin{array}{r}\text { Chl } \\
\text { positiv }\end{array}$ & dia \\
\hline Women & & & \\
\hline $\begin{array}{c}\text { Local: Pelvic inflammatory disease } \\
\text { Bartholinitis/abscess }\end{array}$ & $\begin{array}{l}+ \\
+\end{array}$ & $\begin{array}{l}+ \\
-\end{array}$ & $\begin{array}{l}+ \\
-\end{array}$ \\
\hline $\begin{array}{l}\text { Systemic: Disseminated infection } \\
\text { Men }\end{array}$ & + & 一 & - \\
\hline $\begin{array}{l}\text { Local: Epididymitis/orchitis } \\
\text { Prostatitis ( } \pm \text { vesiculitis) }\end{array}$ & $\begin{array}{l}+ \\
+\end{array}$ & $\begin{array}{l}+ \\
+\end{array}$ & $\begin{array}{l}+ \\
+\end{array}$ \\
\hline $\begin{aligned} \text { Systemic: } & \text { Reiter's disease } \\
& \text { Disseminated infection }\end{aligned}$ & $\overline{+}$ & + & + \\
\hline
\end{tabular}

\section{Local complications: pelvic inflammatory disease}
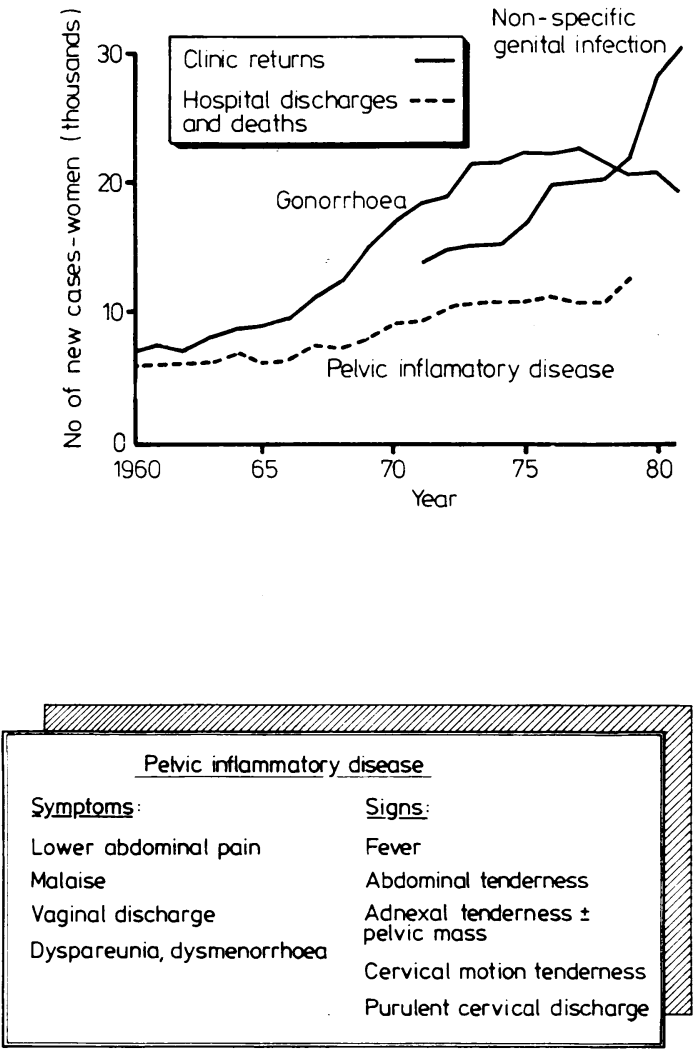

Pelvic inflammatory disease is the most important complication associated with these three types of infection. About $10 \%$ of patients develop this complication after a gonococcal or non-gonococcal infection. In England and Wales the number of cases admitted to hospital has doubled in the past 20 years. In the United States the direct costs of such hospital admissions alone are $\$ 400 \mathrm{~m}$ a year. In some African countries up to $45 \%$ of gynaecological admissions are due to this condition. In all of these countries many more cases are managed on an outpatient basis, for which no routine statistics are available.

Long term morbidity after recovery from acute pelvic inflammatory disease is considerable: chronic abdominal pain, menstrual disturbances, dyspareunia, infertility, tubal pregnancy, medical consultation and medication, time off work, and psychological sequelae. The most disastrous consequence of salpingitis is sterility. The proportion of patients with salpingitis who develop tubal occlusion rises from $10-13 \%$ with a first attack to $75 \%$ with three or more.

The diagnosis of pelvic inflammatory disease is often difficult. Clinically there is a combination of symptoms and signs, but even when these are correlated with laparoscopic findings the clinical diagnosis is correct in only $65 \%$ of patients. The condition is most often confused with appendicitis, endometriosis, and ectopic pregnancy.

Since acute pelvic inflammatory disease is often the direct result of a sexually transmitted infection, full microbiological tests must be carried out to detect infection with Chlamydia trachomatis or Neisseria gonorrhoeae.

In all cases patients should be encouraged to rest in bed, even if they are not admitted to hospital. Any intrauterine device should be removed once treatment has started.

Tetracycline is the drug of choice for either chlamydia positive or chlamydia negative non-gonococcal salpingitis (details are given in the box at the end of the article). Since these two types of infection are often polymicrobial, metronidazole should also be given to eradicate any possible anaerobic infection. If the patient has severe pelvic inflammatory disease- 

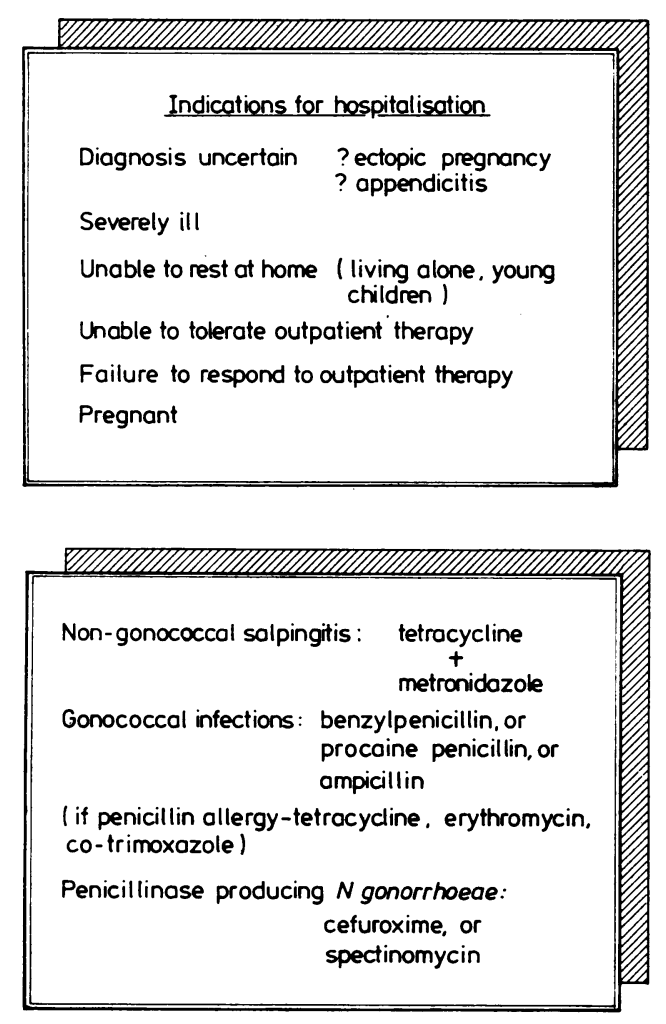

\section{Local complications: vulva}

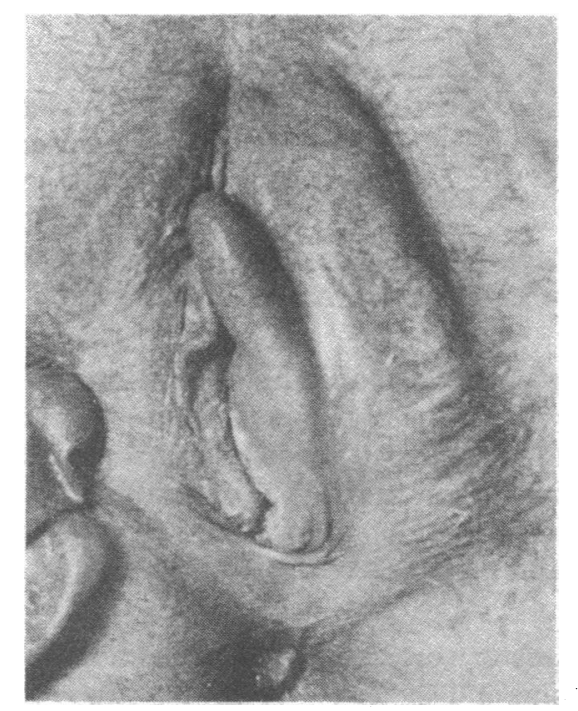

for example, pelvic peritonitis-it may be necessary to start treatment intravenously before using oral regimens.

Gonococcal infections need initial treatment with intramuscular benzylpenicillin or procaine penicillin or oral ampicillin and probenecid followed by ampicillin with probenecid. Alternatively, treatment with intramuscular penicillin may be continued until there are signs of clinical improvement (usually within $\mathbf{4 8}$ hours) before ampicillin is given. Finally, intravenous treatment may be indicated.

Gonococcal and chlamydial infections may occur together, and treatment with penicillin will only partially resolve the salpingitis. In such cases tetracycline should be given with metronidazole. The.US Centers for Disease Control recommend that a broad spectrum combination of antibiotics should be used from the outset to eradicate $N$ gonorrhoeae and $C$ trachomatis with or without anaerobes. Thus examples of inpatient therapy would be intravenous doxycycline and cefoxitin or gentamicin and clindamycin and on an outpatient basis cefoxitin or ampicillin as an immediate dose followed by doxycycline or tetracycline by mouth for 10-14 days. This belt and braces approach is partly dictated by the fact that most patients with pelvic inflammatory disease are treated by private physicians, who often have no special knowledge of sexually acquired disease or access to microbiological support services.

For penicillinase producing strains of $N$ gonorhoeae cefuroxime or spectinomycin is the drug of choice. If a patient with gonococcal salpingitis is allergic to penicillin then tetracycline, erythromycin, or co-trimoxazole may be used.

All sexual contacts of the patient should be traced, to prevent infection of others and reinfection of the patient. Seeing contacts in this way and taking specimens for microbiological tests also help in identifying likely causative organisms which may have been missed in the female partner.

Bartholinitis or Bartholin's abscess is an uncommon complication of gonorrhoea. The patient may present with labial pain and swelling and sometimes difficulty in walking. Early in the condition pus may be visible or may be massaged out of the duct. Once an abscess has formed, however, a fluctuant mass will be felt. Specimens of pus should be collected from the duct (if draining), cervix, and urethra for microscopy and culture to isolate the gonococcus. Cultures for $C$ trachomatis should also be performed since it may coexist with $N$ gonorrhoeae. If $N$ gonorrhoeae is identified and the duct is draining without an abscess having formed the patient should be treated with one intramuscular dose of either benzylpenicillin $(5 \mathrm{Mu})$ or procaine penicillin $(2 \cdot 4 \mathrm{Mu})$, or oral ampicillin $2-3 \mathrm{~g}$ followed by ampicillin $500 \mathrm{mg}$ four times a day for five to seven days. Probenicid should be given in association with penicillin. An abscess will not resolve adequately on antibiotics alone and marsupialisation is necessary to avoid further abscess formation. Sexual contacts should be investigated and urethral tests performed since, as with pelvic inflammatory disease, these often help in identifying the aetiological agent and preventing spread of the disease and reinfection.

\section{Local complications: epididymis and testis}

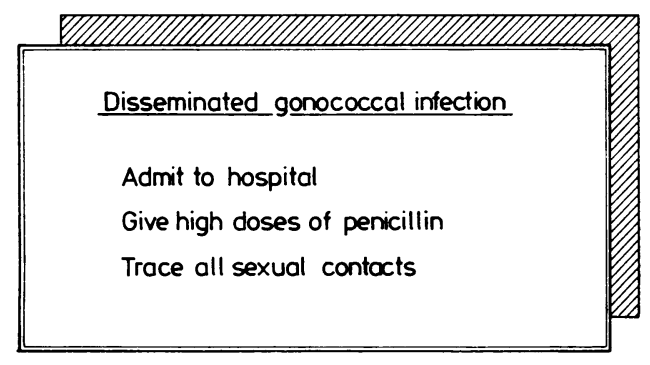

Epididymitis and orchitis due to a sexually transmitted agent are now rare. Patients with either condition or a combination (epididymo-orchitis) may also have a urethral discharge, but this is not invariable. A diagnosis of testicular torsion, urinary tract infection, neoplasm, or tubercle and viral infections affecting the testes (mumps, coxsackie, etc) should not be overlooked.

If gonorrhoea is confirmed by microscopy or culture of a sample of urethral discharge the usual treatment regimen of an initial dose of intramuscular or oral penicillin followed by oral ampicillin and probenecid for seven days should be given. Non-gonococcal infections should be treated with tetracycline $500 \mathrm{mg}$ four times a day for two weeks. A scrotal support should be worn and in severe cases bed rest is necessary. Sexual contact should be seen and investigated for chlamydia and gonorrhoea. 


\section{Local complications: prostate}
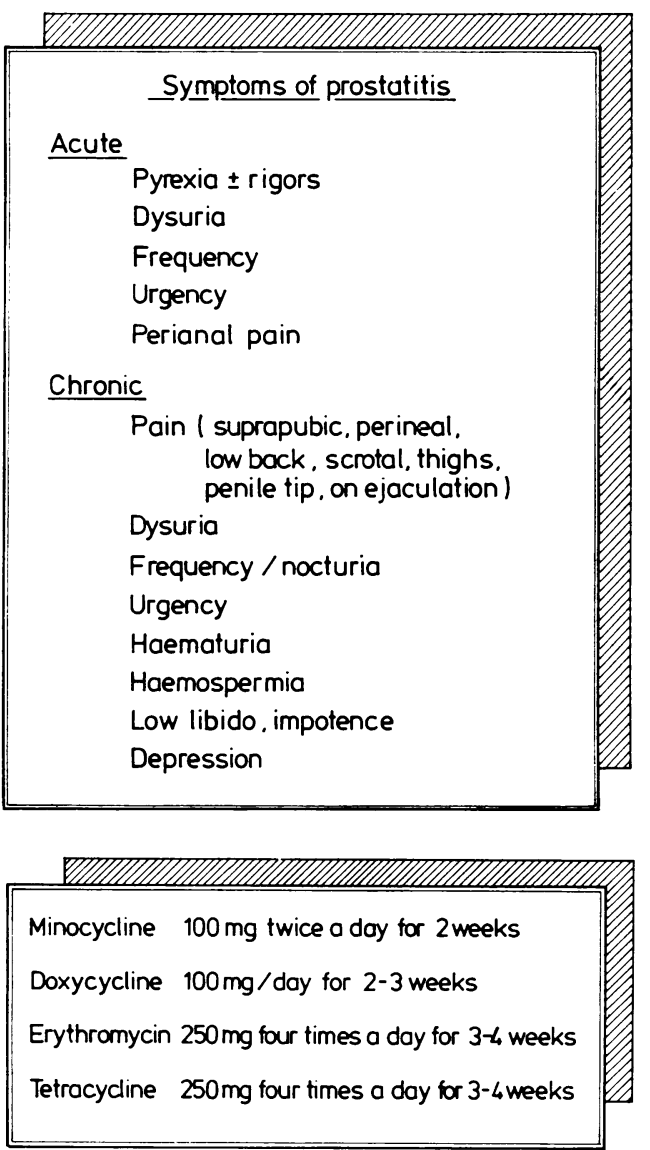

\section{Systemic complications}

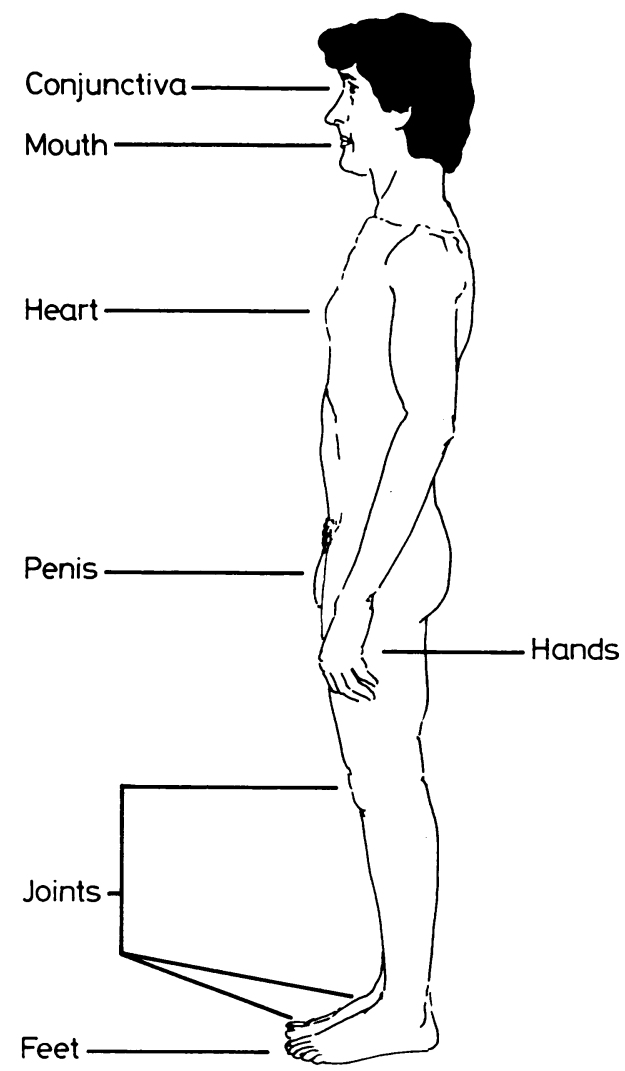

Prostatitis may be associated with gonococcal and non-gonococcal infections as well as with a urinary tract infection. Chronic prostatitis is more common than acute prostatitis. Though rare, prostatitis associated with gonorrhoea is usually acute. In acute disease frequency of micturition, dysuria, urgency, and fever are more common symptoms than pain. On the other hand, in chronic prostatitis, which is usually caused by chlamydia-positive or chlamydia-negative non-gonococcal infections, pain is the most troublesome symptom; it may be suprapubic, perianal, lumbar, or scrotal; affect the thighs or tip of the penis; or be noticed only on ejaculation. Dysuria, frequency of micturition, haemospermia, low libido, impotence, or depression may also occur.

An accurate clinical history, thorough physical examination, and microbiological tests are all important in establishing a diagnosis. The prostate is only rarely tender and enlarged on rectal examination, and this is usually found in association with acute rather than chronic prostatitis. To obtain a specimen of prostatic secretion for microscopy and culture the prostate needs to be massaged. This procedure can precipitate an epididymitis in the presence of a posterior urethritis or cystitis and should not be carried out unless the second of the two glass urine test is clear.

A mid-stream specimen of urine should be obtained for culture. The method used to obtain a specimen of prostatic fluid varies from straightforward massage to the Stamey technique of collecting urine both before and after the massage to differentiate between prostatitis, cystitis, and urethritis. More than 10 leucocytes per high power field $(\times 40$ magnification) or clumping of the cells, or both, in the prostatic secretion will indicate prostatitis. Further investigations of the urinary tract are indicated if a urinary tract infection is discovered.

If no organisms are identified on microscopy or culture of the prostatic fluid a broad spectrum antibiotic which is lipid soluble and so diffuses into the prostate should be given - for example, minocycline, doxycycline, or erythromycin. Tetracycline may be given, but it is less effective as it does not penetrate the prostate well.

Occasionally patients may present with or develop two rare but serious complications affecting multiple sites.

\section{Reiter's disease}

Reiter's disease, which is a seronegative polyarthropathy occurring as a rare $(0 \cdot 5-1 \%)$ complication of non-gonococcal urethritis, affects mainly men. $C$ trachomatis, Shigella, Salmonella, and Yersinia have also been implicated in the aetiology of this condition. Conjunctivitis may also occur with the urethritis and arthritis but it is not an essential feature of the condition. The leg joints, particularly knees, ankles, and feet, are those most commonly affectec'. Other manifestations are mucous membrane lesions of the mouth and penis (circinate balanitis), keratodermia blenorrhagica of the feet and hands, onycholysis and ridging of the nails, and, rarely, pericarditis, partial heart block, aortic incompetence, peripheral neuropathy, and meningoencephalitis. An accurate clinical history is essential to exclude other arthropathies, such as rheumatoid arthritis and ankylosing spondylitis and those occurring with psoriasis, Crohn's disease, and ulcerative colitis.

Non-steroidal anti-inflammatory agents are given in mild cases (indomethacin $25 \mathrm{mg}$ three times a day or phenylbutazone $100 \mathrm{mg}$ three times a day). Bed rest, but not total immobilisation, is recommended during the active phase of the disease. Systemic steroids should be reserved for seriously ill patients or for those with complications such as pericarditis and uveitis. A concurrent non-gonococcal urethritis should be treated with tetracycline. The prognosis of Reiter's disease is extremely variable and relapses do occur. 


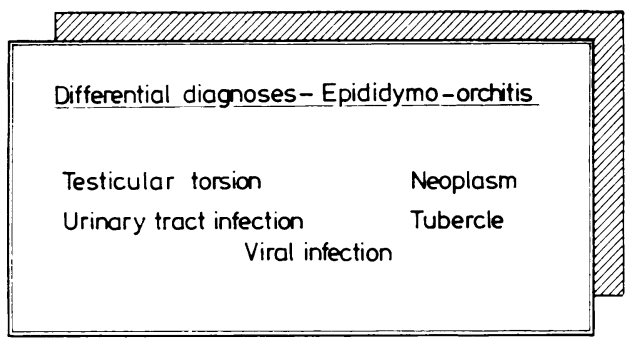

\section{Disseminated gonococcal infection}

Disseminated gonococcal infection is a rare complication of gonorrhoea and affects women more often than men. The common symptoms are pain in the joints (wrists, knees, elbows, ankles, or small joints of the hand), tenosynovitis, and rash. The initial erythematous macular papular lesions develop into frank vesicles and pustules; they appear as crops on the trunk and arms and legs and often in association with a fever. Occasionally endocarditis, myocarditis, pericarditis, and meningitis may occur. Gonococci are often isolated from the genital tract and occasionally from blood and joint fluid but rarely from skin lesions. Patients need to be admitted to hospital and treated with high doses of penicillin. All sexual contacts must be traced.

\section{Infections in other sites}

Rectum - The rectum may be affected in several sexually transmitted conditions (gonorrhoea, chlamydia, non-gonococcal infection, genital herpes and warts, syphilis, Entamoeba histolytica, Giardia lamblia, and trauma). Only gonorrhoea is considered in this article. Rectal gonorrhoea is often symptomless and illustrates the need for contact tracing and regular check ups for homosexuals who are having casual sexual encounters. Symptoms, when they occur, are anal discomfort and pain, painful defecation, and a blood stained or purulent rectal discharge. Diagnosis is by microscopy and culture. Microscopy is incorrect in half of all cases since it is difficult to identify gonococci in the presence of many other organisms in the rectum. Cultures must always be performed if rectal gonorrhoea is suspected. Treatment and follow up of rectal gonorrhoea are the same as those for uncomplicated urethral or cervical infections.

Throat-Even though still uncommon, oropharyngeal gonorrhoea is being seen more often and may be asymptomatic. Patients admitting to orogenital contact (particularly homosexuals) will have throat swabs taken in a clinic in an attempt to isolate $N$ gonorrhoeae. Patients with infection of the throat but no other site may also infect their partner's urethra during fellatio. Diagnosis is by culture; microscopy is useless owing to the presence of mixed organisms, particularly commensal neisseria. In patients with pharyngeal infection high doses of penicillin or other antibiotics are needed to eradicate the organism. Ampicillin 3 $\mathrm{g}$ plus $1 \mathrm{~g}$ probenicid followed by $500 \mathrm{mg}$ ampicillin four times a day for three days or co-trimoxazole (two tablets three times a day for seven days) are recommended treatments.

Professor Michael W Adler, MD, MRCP, is professor of genitourinary medicine, Academic Department of Genitourinary Medicine, Middlesex Hospital Medical School, London.

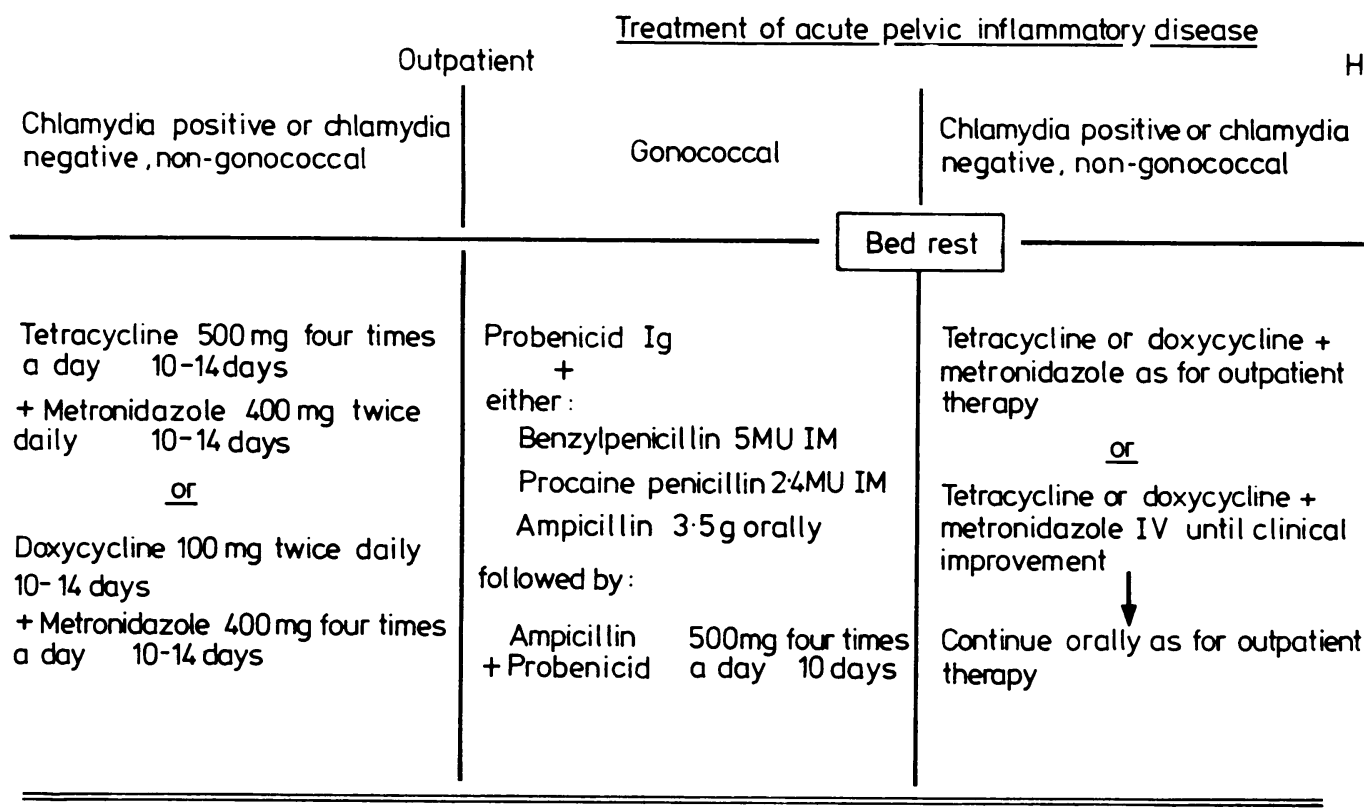

1. Penicillinase producing $N$ gonorrhoeae

2 Patients allergic to penicillin, with gonococcal pelvic inflammatory disease improvement
Spectinomycin or cefuroxime $2.0 \mathrm{~g}$ IM three times a day until clinical

Tetracycline $500 \mathrm{mg}$ four times a day 10-14 days

Erythromycin stereate $500 \mathrm{mg}$ four times a day 10-14 days

Co-trimoxazole 4 tablets twice daily

7-10 days

Spectinomycin or cefuroxime as above
Benzylpenicillin or procaine penicillin or ampicillin followed by ampicillin as for outpatient therapy

\section{or}

Benzylpenicillin 5MU IM + probenecid $1 \mathrm{~g}$ orally followed by $\downarrow$ Benzylpenicillin 1MU IM four times a day + probenecid $500 \mathrm{mg}$ orally until clinical improvement Ampicillin $\$ 500 \mathrm{mg}$ four times + Probenecid $\}$ a day 10 days 으

Benzylpenicillin, ampicillin, or amoxycillin IV until clinical improvement

continue orally 